\title{
Finite element analysis of aluminum-Kevlar/Epoxy pressure vessel
}

\begin{abstract}
In this present work, the composite pressure vessel type three has been investigated by finite element method (FEM). The aluminum pressure vessel reinforced with Kevlar/Epoxy (Aramid 149) was analyzed under internal pressure to predict the ultimate failure pressure of the vessel. Also the optimum winding angle which provides the highest strength for the vessel was determined by applying Tsai-Wu and Tsai-Hill failure theories. The asymmetric fiber orientation for six different winding angles was utilized to reinforce the aluminum vessel. The commercial code ABAQUS/CAE was employed to analyze the composite vessel. Results obtained from the simulation were in good consistency with the analytical and the experimental outcomes.
\end{abstract}

Keyword: Composite pressure vessel; Failure pressure; Finite element analysis; Winding angle 